Rev. salud pública. 14 sup (2): 129-141, 2012

\title{
Motivar el cambio a partir de la educación para la salud
}

\author{
Motivating health education-based change
}

Ana H. Puerto-Guerrero

Facultad de Enfermería, Universidad Nacional de Colombia. ahpuertog@unal.edu.co

Recibido 25 Mayo 2011/Enviado para Modificación 8 Agosto 2011/Aceptado 3 Noviembre 2011

\section{RESUMEN}

Se hace referencia a una experiencia académica en la formación de profesionales de enfermería, en las asignaturas: Prevención primaria y Enfermería del niño del área de Salud Pública. A partir de la sistematización de las experiencias acumuladas se procedió a identificar los elementos epistemológicos, teóricos y prácticos de la propuesta que se desarrollo durante cinco años en Instituciones educativas en el nivel de básica primaria, con la propuesta educativa "Vivir la sexualidad con dignidad" se encuentra que este tipo de trabajos lograr desarrollar una motivación especial en la comunidad escolar al tiempo que permite a los universitarios acercarse a las realidades sociales que enfrentaran en su práctica profesional. Se insiste en la necesidad de fortalecer en los niños la posibilidad de participar de la familia, el estado y la sociedad civil.

Palabras Clave: Niño, salud pública, educación en enfermería, salud escolar, educación sexual (fuente: DeCS, BIREME).

\section{ABSTRACT}

The following work refers to academic experience regarding the training of nurses concerning primary prevention and child nursing within the area of public health. The target population consisted of children. Accumulated experience was systematised for identifying epistemological, theoretical and practical elements developed over five years in basic primary education institutions, in line with the educational proposal Experience sexuality with dignity. It was found that this type of work managed to develop special motivation in the scholastic community whilst allowing academics to approach the social reality which they must confront in their professional practice. The work emphasised strengthening children's awareness of the need for family, state and civil society participation. Motivating health education-based change did involve the systematisation of experience as a methodological tool.

Key Words: Child, education, sexuality, nursing, public health (source: MeSH, NLM). 
E l mejoramiento continuo en la formación de los profesionales de la salud requiere de los docentes una reflexión continua alrededor de la manera en la que se desarrolla el proceso y la proyección de los discentes durante su práctica; en este proceso resulta pertinente la retroalimentación que se puede lograr tanto del ejercicio profesional de los egresados y los profesores como de los campos de práctica en los que se realiza el entrenamiento.

Este trabajo pretende aprovechar las experiencias adquiridas en la actividad profesional y docente en salud pública y que han estado orientadas a la protección de la niñez, se efectúa una sistematización de las mismas señalando los marcos epistémicos, teóricos y prácticos que dentro de un contexto socioeconómico y político, permiten aproximarse a la comprensión de la convocatoria a trabajar por la niñez, las etapas de desarrollo de la experiencia, los elementos teóricos y metodológicos y la propuesta educativa que hacia el niño y la niña escolarizados se deriva, a partir de la recuperación de la experiencia sobre el Programa de Educación Sexual denominado "Vivir la sexualidad con dignidad" desarrollado en dos instituciones educativas en un municipio de Cundinamarca.

\section{La Universidad Nacional de Colombia (1) incluye entre sus propósitos:}

"Formar profesionales e investigadores sobre una base científica, ética y humanística, dotándolos de una conciencia crítica, de manera que les permita actuar responsablemente frente a los requerimientos y tendencias del mundo contemporáneo y liderar creativamente procesos de cambio; Formar ciudadanos libres y promover valores democráticos, de tolerancia y de compromiso con los deberes civiles y los derechos humanos; Estudiar y analizar los problemas nacionales y proponer, con independencia, formulaciones y soluciones pertinentes; Hacer partícipes de los beneficios de su actividad académica e investigativa a los sectores sociales que conforman la nación colombiana".

En este contexto desde la década de los 80 se incluyó en el plan de formación de enfermeros la asignatura enfermería en prevención primaria, cuyo enfoque se sustentó en la Atención Primaria en Salud-APS y con la reforma de 1992 se incluyó enfermería del niño, con un carácter de práctica social orientada a la protección de la vida y la salud de la niñez, esta vez sustentada en el proceso vital humano, su ciclo vital y el cuidado de enfermería. En ambos casos el escenario fueron los servicios ambulatorios y comunitarios y el propósito fue la comprensión de la Salud Pública, en dos de sus dimensiones: 
Como un saber matizado por “...contenidos ideológicos, científicos y tecnológicos determinados por las condiciones históricas que se han impuesto al conocimiento y su transmisión a lo largo del tiempo" (2), que por razones de ética social debe llegar a toda la población con principios de equidad, igualdad, justicia y respeto por los derechos humanos en la que el profesional se esfuerza para que dicha creencia se exprese en acciones, a través de una correcta aplicación de las bases científica y técnica de esta disciplina (3), aspecto que resulta consecuente con los deberes legales de los profesionales de enfermería (4) y que resulta de especial interés dado el enfoque biologista y medicalizado, que desconoce la existencia de los determinantes sociales que inciden en la cotidianidad de las personas y los colectivos.

Como práctica social o quehacer que demanda una intencionalidad, un objeto de interés y unos métodos, que pretender dar respuesta a unas necesidades concretas del ser humano (6) y que se enmarca en una conceptualización concreta acerca de su función esencial (7), para lo cual, en el caso de los profesionales de enfermería, se recurre a herramientas como el proceso de atención de enfermería, el proceso administrativo y el proceso epidemiológico e investigativo, las cuales se aplican en los ámbitos donde la persona vive, trabaja, estudia, se recrea y se desarrolla, así como en las instituciones que directa o indirectamente ofertan servicios de Salud (4).

El enfoque en la población infantil se considera pertinente por la desventaja que para exigir sus derechos y libertad implica su alta vulnerabilidad (8), y se desarrollo en el contexto de los programas: Supervivir, Salud Escolar y Escuela saludable (9), teniendo presente las dinámicas de la política pública y normas nacionales e internacionales, relacionadas con la niñez y los espacios que ofrecen la Salud y la Educación y apalancándose en la participación en programas orientados a "Desarrollar una sana sexualidad que promueva el conocimiento de sí mismo y la autoestima, la construcción de la identidad sexual dentro del respeto por la equidad de los sexos, la afectividad, el respeto mutuo y prepararse para una vida familiar armónica y responsable” (10), eligiendo como enfoque epistemológico la educación problematizadora (11), que implica la lectura de la realidad, en el contexto de un devenir histórico construido por las personas y los colectivos con el fin de generar procesos de transformación de esa realidad, mediante el diálogo y el encuentro entre las personas, que exige desarrollar la capacidad de escucha, respeto, y solidaridad. 
La experiencia académica, que fue realizada por estudiantes y docente, se expresó en los dos ejes del proyecto: La salud y la educación, en el primero se inició con el diagnóstico situacional seguido de acciones de promoción de la salud orientadas a motivar la participación social de la comunidad educativa y el desarrollo de las políticas y planes definidos en esos momentos por el sector educativo y el sector salud, se tuvo un especial énfasis en la participación de los niños y las niñas reconociéndolos como sujetos de derechos, con capacidades y potencialidades que debían ser desarrolladas para trascender la etapa de desarrollo convencional y alcanzar su autonomía (12). La educación se abordó la institución con la intención de convertirla en un ambiente favorable y digno dentro de la estrategia de "Escuela Saludable" teniendo como componentes: Formulación y aplicación de políticas escolares saludables; Creación y conservación de ambientes escolares saludables; Educaciónpara la salud con enfoqueintegral; Fortalecimiento de laparticipación social y comunitaria y Servicios escolares de salud, nutrición y vida activa, a partir de la conceptualización de que los niños requieren atención y protección destacando la responsabilidad de la familia en materia de atención primaria y protección (13). Al respecto se sabe que la educación es un proceso continuo y permanente a través de la vida, que inicia en el seno familiar y continua en los jardines infantiles para luego llegar a la escuela, espacio de interés para este trabajo puesto que allí se desarrollan los procesos psicológicos superiores: Toma de decisiones, lenguaje, pensamiento y abstracción, orientados al conocimiento y dominio progresivo de las herramientas culturales y el control de su propio comportamiento (14).

El proceso se desarrollo en tres etapas: Acercamiento, que corresponde a la fase de observación, indagación e identificación de los espacios de trabajo, las cuales están mediadas por experiencias previas, poderes, valores y prevenciones, que definen los acuerdos para iniciar el proyecto educativo; Luego Sensibilización: que se relaciona con el proceso persuasivo para que los integrantes de la comunidad educativa acepten la propuesta, en este momento se buscó incidir en lo emocional, lo cognitivo y lo socio-afectivo para que internalizaran la importancia de la propuesta, buscando lograr un proceso participativo de negociación y concertación, de construcción y reconstrucción y finalmente el cumplimiento de las acciones, para lo cual se inició el proceso con el juego asociativo, con el fin de integrar el profesional de enfermería en formación como facilitadores y los niños y las niñas; se buscó conocer intereses, actitudes, capacidad de vincularse con otros/otras, también se observó el compartir, la vivencia de los valores, la diferencia 
entre ellos y ellas, conflictos que pudieran surgir y alternativas que ellos y ellas encontraran para superarlos. Posteriormente al definirse el tema se desarrollaron juegos individuales, cooperativos, competitivos de acuerdo al objetivo propuesto, para lo cual también se contó con la creatividad de los participantes para proponer los argumentos que sustentaron la utilidad de los títeres y los sociodramas, que así como el cuento y las narrativas, fueron otras estrategias de aprendizaje.

Aunque hubo oportunidad de fortalecer diversas áreas como el desarrollo del lenguaje, el desarrollo motriz y el desarrollo social, entre otros, el primer logro de este trabajo tiene relación con la motivación de los agentes de los procesos educativos (profesores, niños, adolescentes, padres y practicantes), expresada en:

La integración generada a partir de la reflexión y el dialogo acerca del estilo de vida, lo cual se convirtió en una medio para la comprensión de la importancia de tener una vida saludable.

La participación en el juego intencionado, mediante el cual se aprende con alegría y se genera un clima de armonía y creatividad, estimulando la capacidad para ver al otro y por ende el respeto y solidaridad entre otros valores.

La comprensión de la práctica profesional de enfermería, como disciplina social que requiere de estas estrategias pedagógicas puesto que todo acto de cuidado con la persona, familia, colectivo o comunidad, lleva implícita la acción educativa.

La receptividad que tuvieron los estudiantes de enfermería, que se convirtió en un hecho facilitador para el proceso educativo con la niñez, eventualmente asociado a que se trata de jóvenes lo cual podría estar favoreciendo el desarrollo de confianza e interés por su labor.

Se considera que la metodología de: "Nadie educa a nadie, nadie se educa a sí mismo; Las personas se educan entre sí, mediatizados por el entorno”, está fuertemente implicada en este aspecto. También se destaca la importancia dada al respeto por los valores culturales de las comunidades y el papel fundamental de la cooperación cuando se trata de garantizar los derechos de los niños y las niñas (15), en cuanto al primero resulto necesario estudiar los 
determinantes estructurales e intermedios con el fin de motivar un cambio en las actitudes de quienes los cuidan y protegen, dicho cambio se orientó al deber de reconocerlos y respetarlos como sujetos sociales, con capacidades y habilidades que se van desarrollando según procesos psicológicos superiores (14), en cuanto al segundo se buscó lograr una interacción social rica en experiencias que les facilitaran internalizar unas prácticas cotidianas de socialización y que les proveyeran de vivencias para impulsar su potencial y enriquecer el avance de sus capacidades intelectuales, sociales y políticas, entre otras. Igualmente se considera esencial el trabajo de los aspectos éticos y morales que, aunque tienen una profunda relación con el sistema social en el que se encuentran, deben enmarcarse dentro de los mínimos socialmente aceptables (16) y que señalan la importancia de avanzar en los aspectos de autonomía, concientizándose de los criterios que les guían y ubican en una realidad que les ofrece diferentes opciones de vida, debiendo recurrir al discernimiento acerca de la conveniencia de una decisión y las consecuencias que al tomarla o no deben asumir.

Un segundo producto importante es el que tiene que ver con el desarrollo de habilidades y conocimientos de los estudiantes de enfermería con respecto al cuidado de la vida y la salud (17), entendida esta última como un bien interno, de construcción histórica, esencia y objeto lógico de la práctica social que se origina en la intercomunicación entre el profesional de enfermería (cuidador) y la persona o grupo sujeto de cuidado. Al respecto el aprendizaje compartido tiene relación con principios como individualidad, integralidad, dialogicidad, continuidad, calidad y oportunidad, con fines como el ayudar a desarrollar al máximo los potenciales individuales y colectivos y mantener niveles óptimos de prácticas de vida saludables con el propósito de promover la salud, prevenir la enfermedad, intervenir en el tratamiento, rehabilitación y recuperación de la salud, aliviar el dolor, proporcionar medidas de bienestar y contribuir a una vida digna de la persona, para lo cual se utilizan el método administrativo gerencial y los procesos de enfermería y epidemiológico.

El tercer alcance de las actividades tiene que ver con el organizar los espacios de trabajo con la niñez de kínder a quinto de primaria en el desarrollo de un programa de educación para la Salud, basado en el modelo de desarrollo humano, lo cual permitió y facilitó formular una nueva propuesta de Educación Sexual: "Vivir la sexualidad con dignidad”, que se enmarcó en el lineamiento filosófico y ético del proyecto nacional de educación sexual para la vida y el amor (18). Al respecto es importante la confluencia 
de intereses entre la formación básica primaria y el rol comunitario del trabajo en enfermería, en el primero se incluye la formación de los valores fundamentales para la convivencia en una sociedad democrática, participativa y pluralista (10), incluyendo la valoración de la higiene y la salud del propio cuerpo, la formación para la protección de la naturaleza y el ambiente, la formación para la participación y organización, la utilización adecuada del tiempo libre, el desarrollo de valores civiles, éticos, morales, de organización social y de convivencia humana y la adquisición de habilidades para desempeñarse con autonomía en la sociedad. En el segundo la enfermería como profesión liberal y disciplina social sustenta sus conocimientos en las ciencias biológicas, sociales y humanísticas y en sus propias teorías, conocimientos y tecnologías que analizados y asimilados críticamente se convierten en sustentos para comprender la vida y la salud de las personas en lo individual y en lo colectivo y argumentar los determinantes sociales que inciden en la cotidianidad del niño y la niña, por esto se logro que de manera sinérgica se confrontaran el saber popular con el conocimiento científico y la experiencia de vida y salud cotidiana con la tradición educativa. Estas confrontaciones condujeron a procesos de aprendizaje y desaprendizaje a partir de espacios reflexivos sobre los derechos, en particular acerca de la salud, la educación, la participación y la información, entre otros.

La cuarta comprensión emanada del proyecto es la que tiene que ver con la familia, la cual “...no sólo es el espacio de producción y reproducción, sino que es el ámbito primario donde se construye la socialización, las relaciones familiares y la organización económica, por medio de la interacción conjunta de los integrantes, quienes poseen habilidades y capacidades para contribuir a la transformación sociocultural-económica y política" (19) y a su vez es “...una institución social mediadora entre lo público y lo privado; entre la dimensión biológica de la reproducción de la especie y la función cultural de interiorizar en los individuos las normas, los valores que les permiten convivir en sociedad"(20), esta institución, por la dinámica en la cual ha estado inmersa, ha vivido transformaciones como el haber pasado de familias extensas a familias nucleares, la vinculación de la mujer al mercado laboral que hizo que se convirtiera en muchos casos en la principal proveedora, generándose tensiones entre las parejas por cuestiones de autoridad y poder, la protección de los hijos por parte de terceras personasfamiliares o no- y finalmente la crisis económica que causó bajos ingresos y por ende bajas coberturas en salud, difícil acceso a la educación y falta de continuidad en las propuestas educativas que se llevaban de otros sectores, en este caso, de la facultad de enfermería.

Otros hallazgos están relacionados con situaciones particulares que 
expresan la vulnerabilidad de este grupo, tales como el difícil acceso a la escuela, las limitaciones sensoriales, el acceso al consumo de sustancias psicoactivas, alcohol y tabaco, la inserción laboral precoz, la exposición a ambientes inseguros y la inequidad en el acceso a servicios públicos, frente al cual la saludes vista como "...un proceso que posibilita el desarrollo del proyecto vital humano, de las comunidades y de la sociedad en general. La realización cotidiana del proyecto de vida de los sujetos, entendida como una construcción intencionada y determinada por las condiciones sociales, económicas y políticas, así como por las respuestas sociales organizadas" (21) y el desarrollo como "...un proceso de expansión de libertades reales de que disfrutan los individuos" (22), que confluyen en las actividades de Promoción de la Salud, campo en el que los derechos individuales y colectivos, como condiciones de vida, constituyen un marco de referencia en la cotidianidad de las personas, en el que igualmente existen deberes, cuya permanente interrelación permite la construcción de los proyectos de vida; En este sentido el trabajo buscó mejorar la conciencia de los determinantes sociales para participar en la definición de políticas públicas, para eliminar las inequidades y la exclusión social y tener acceso a programas que potencialicen el desarrollo de las capacidades y la autonomía para la libertad, mejorando la exigibilidad de los derechos y como clave de ellos, la participación en espacios de decisión en los que se requiere la formulación de propuestas que se sustentan en problemáticas socioeconómicas y políticas, reflejadas en inequidades sociales que conducen a la exclusión, la pobreza, el desempleo, la violencia, la enfermedad y la muerte.

La estrategia educativa busco incidir en las cinco esferas que conforman el concepto de calidad de vida: La persona como ser individual autónomo que toma decisiones y desarrolla sus capacidades para aprender a comprometerse, ser responsable y generar acciones en la búsqueda de satisfactores, dentro de un contexto social; La equidad, que pretende la mayor comprensión de la realidad propia y circundante, a fin de identificar cuáles son las posibilidades de ejercer la autonomía en los diferentes espacios de interrelación, tanto institucionales como colectivos; La institucionalidad como forma de organización y participación de los diferentes sectores y colectivos y sus relaciones internas y externas; La relación con el medio ambiente y finalmente la subjetivación. En el ejercicio de los profesionales del sector la educación para la salud puede señalarse como un satisfactor de las necesidades axiológicas de protección, subsistencia, participación, entendimiento, creación e identidad y de las necesidades existenciales de ser, tener, hacer y estar (6). Tener acceso a la educación es un derecho, y es deber de las personas y los colectivos, acceder a ella, para aprender y aprehender, como se cuida la vida y la salud y cuál es la importancia de estar 
en espacios de socialización con el fin de compartir con otros las costumbres, valores, mitos y creencias, sobre las formas de cuidarse y proteger la vida.

Algunas dificultades en el proceso tuvieron que ver con una cierta incomprensión de la educación problematizadora y rol del juego en la misma, del cual se afirmaba que era contraproducente por el "desorden que hacían", porque "no se transmitía la seriedad que implicaba el proceso", por los "tiempos" que se utilizaban; porque "todos quieren hablar a la vez", porque los niños "se ríen demasiado", porque "con tanta paradera, se distraen y no se quedan quietos", expresiones relativamente frecuentes de los docentes quienes aún no reconocían en la práctica el derecho a la participación de los niños y las niñas y al disfrute de una actividad lúdica que les facilitaba expresar lo que sabían, de quienes lo habían aprendido y los valores que estaban implícitos en esos saberes.

A este respecto es importante destacar que si bien cierto que el juego es considerado como una actividad de entretenimiento, también es una forma de interactuar en cualquier época de la vida que exige responsabilidad y compromiso y que en la niñez hay especial motivación e interés por el mismo, lo que genera sentimientos de goce que se expresan participando propositivamente, de tal manera que el juego se convierte en el espacio donde se pueden identificar los valores que lo mediatizan, se potencializa el desarrollo de las capacidades intelectuales y de interacción (ejercicio de la ciudadanía), se facilitan las expresiones culturales y políticas; se favorece el desarrollo de comportamientos morales; se proporciona seguridad y se dispone al niño para afrontar la resolución de conflictos. Es por esto que el juego como estrategia pedagógica facilita la problematización de la realidad y por ende provee la comprensión de experiencias, vivencias y lenguaje, convirtiéndose en juego didáctico. Así pues en esta propuesta el juego se convirtió en la mejor herramienta para prescindir de miedos al hablar de la sexualidad con la niñez y para cambiar expresiones lingüísticas que conducían a la risa maliciosa producto de concepciones erróneas sobre la temática

Otros desencuentros se dieron por las creencias, los mitos, tabús, temores y miedos asociados a la temática elegida y al enfoque integral de la sexualidad y no sólo de la genitalidad que como consecuencia llevo a que se abordaban aspectos como la prevención del embarazo y de las infecciones de transmisión sexual, sin embargo la comprensión de que "la sexualidad está presente a lo largo de la vida de todos los seres humanos y su desarrollo armónico es fundamental para la formación integral de la persona. Su finalidad es la relación humana, 
en tanto que contempla dimensiones comunicativas, afectivas, de placer y reproductivas y su desarrollo comprende aspectos biológicos, psicosociales, culturales y éticos” (18), razón por la cual la educación sexual debe ser “....impartida en cada caso de acuerdo con las necesidades psíquicas, físicas y afectivas de los educandos según la edad" (10). Esta dificultad se enfrento explicitando los objetivos de: 1. Propiciar cambios en el conocimiento, actitudes y comportamientos relativos a la sexualidad, de acuerdo con la ciencia y el humanismo y fomentar la salud en general y la salud sexual en particular, guardando el respeto que merecen las creencias y valores populares. 2. Replantear los roles sexuales tradicionales, buscando una mejor relación Hombre- mujer que permita la desaparición del sometimiento del uno por el otro, basados en los principios de igualdad social, jurídica y económica de ambos sexos. 3. Promover modificaciones de la vieja estructura familiar, con el fin de buscar una mayor equidad en las relaciones entre padres e hijos y entre la pareja conyugal, propiciando el amor, el respeto, el crecimiento y la autodeterminación de los miembros de la familia. 4. Lograr que de una manera consiente y responsable hombres y mujeres decidan cuál es el momento en que pueden traer hijos al mundo, utilizando adecuadamente los diversos medios de regulación de la fertilidad, y; 5. Fomentar la salud sexual y reproductiva de los educandos tanto físicos como mentales.

Aunque el Ministerio de Educación Nacional, para abordar el tema de la Sexualidad definió cuatro categorías: Persona, pareja, familia y sociedad y para cada grado escolar, desde kínder a once, definió como temas: Identidad, reconocimiento, tolerancia, reciprocidad, ternura, diálogo, cambio, amor y sexualidad, responsabilidad, conciencia crítica y creatividad, la propuesta se convirtió en otra manera de orientar los derechos de la niñez, especialmente el derecho a la libertad de expresión; el cual incluye la posibilidad de "buscar, recibir y difundir informaciones e ideas de todo tipo, sin consideración de fronteras, ya sea oralmente, por escrito o impresas, en forma artística o por cualquier otro medio elegido por el niño", puesto que la exigibilidad de este derecho motiva y facilita la participación expresando sus ideas, experiencias y conocimiento con tranquilidad y autonomía y muestra a través de esa participación, su proceso de desarrollo y su grado de seguridad.

De igual manera los niños y las niñas aprendieron a identificar situaciones peligrosas relacionadas con abusos con el fin de protegerse y perder el miedo a denunciar.

Los contenidos que se desarrollaron buscaron: Facilitar el conocimiento 
integral del Ser Humano a partir de las realidades de los participantes. Indagar la importancia del reconocimiento de la persona como ser único e irrepetible.

Cuadro 1. Enfoque asumido en la propuesta educativa "Vivir la sexualidad con dignidad"

\begin{tabular}{|c|c|c|}
\hline Ideas Centrales & & Puntos a Destacar \\
\hline \multirow{7}{*}{ Identidad } & Soy & $\begin{array}{l}\text { Un ser: Biológico, psicológico, social, político, ético, } \\
\text { estético, histórico, espiritual, cultural y productivo }\end{array}$ \\
\hline & Conocimiento del cuerpo & $\begin{array}{l}\text { Soy Mujer, soy hombre, las partes del cuerpo, } \\
\text { intimidad de mi cuerpo, protejo mi intimidad }\end{array}$ \\
\hline & Sentimientos & Afecto, amor, tristeza, miedo, enfado, alegría, odio \\
\hline & & Enojo, mal genio, resentimiento; Disfrute, alegría, \\
\hline & Emociones & $\begin{array}{l}\text { placer, orgullo; Ansiedad, terror desconfianza, pavor; } \\
\text { Desesperanza, soledad, nostalgia }\end{array}$ \\
\hline & Gustos & $\begin{array}{l}\text { Identificación de gustos; Aspectos positivos y negativos } \\
\text { de los gustos identificados. }\end{array}$ \\
\hline & Sensaciones & Impresiones y reacciones a partir de las percepciones \\
\hline \multirow{4}{*}{ Reconocimiento } & Soy un Ser singular & Soy único e irrepetible; Individualidad; Historia de vida \\
\hline & $\begin{array}{l}\text { Reconozco y expreso mis } \\
\text { emociones }\end{array}$ & $\begin{array}{l}\text { Identificación de la emoción; Libertad de la expresión } \\
\text { emocional; valores en la expresión de las emociones. }\end{array}$ \\
\hline & Reconozco mi cuerpo. & $\begin{array}{l}\text { Características individuales del cuerpo; Respeto por el } \\
\text { cuerpo, protección del cuerpo }\end{array}$ \\
\hline & $\begin{array}{l}\text { Reconozco mis } \\
\text { capacidades. }\end{array}$ & $\begin{array}{l}\text { Exploración e identificación de capacidades; acciones } \\
\text { para potenciar las capacidades }\end{array}$ \\
\hline \multirow{4}{*}{ Tolerancia } & Significado de tolerancia & $\begin{array}{l}\text { Qué es la tolerancia; Importancia de la tolerancia en la } \\
\text { interacción cotidiana. }\end{array}$ \\
\hline & $\begin{array}{l}\text { Significado de la igualdad } \\
\text { y la diferencia }\end{array}$ & $\begin{array}{l}\text { Conceptos de igualdad y diferencia; Importancia de } \\
\text { vivenciar la igualdad y la diferencia en la cotidianidad; } \\
\text { repercusiones de vivir la desigualdad y la intolerancia }\end{array}$ \\
\hline & Aceptación de mi cuerpo & Mi cuerpo es así, quiero mi cuerpo y lo cuido \\
\hline & Respeto & $\begin{array}{l}\text { Qué es le respeto; como me respeto, ventajas de vivir } \\
\text { el respeto }\end{array}$ \\
\hline Reciprocidad & $\begin{array}{l}\text { Sentido y significado de } \\
\text { reciprocidad }\end{array}$ & $\begin{array}{l}\text { Concepto de reciprocidad; Importancia de vivenciar la } \\
\text { reciprocidad; espacios de vivencias de la reciprocidad, } \\
\text { diferenciar la reciprocidad del utilitarismo. }\end{array}$ \\
\hline \multirow{3}{*}{ Vida } & $\begin{array}{l}\text { Sentido y significado de } \\
\text { la vida. } \\
\text { Mi ubicación en la vida }\end{array}$ & $\begin{array}{l}\text { Concepto, la vida como derecho; Importancia de vivir } \\
\text { dignamente } \\
\text { Mis espacios: hogar, las Instituciones; El deambular } \\
\text { con responsabilidad. }\end{array}$ \\
\hline & Cambios en mi Vida. & $\begin{array}{l}\text { Mis transformaciones vividas a través de la Vida, } \\
\text { Historia de vida }\end{array}$ \\
\hline & Para donde voy en la vida & $\begin{array}{l}\text { Proyecto de vida: donde estoy y para donde voy, mis } \\
\text { capacidades y potencialidades; debilidades }\end{array}$ \\
\hline Ternura & $\begin{array}{l}\text { Sentido y significado de la } \\
\text { ternura }\end{array}$ & $\begin{array}{l}\text { Concepto de la ternura, expresiones de ternura; el } \\
\text { respeto en la expresión de la ternura; sentimientos } \\
\text { que se generan cuando se expresa y nos expresan } \\
\text { la ternura; aceptación o rechazo de expresiones de } \\
\text { ternura. }\end{array}$ \\
\hline
\end{tabular}


Reconocer el valor de la tolerancia para generar estrategias de convivencia armoniosa. Identificar la importancia de la reciprocidad en la interacción cotidiana. Reconocer la vida como un proceso y como un derecho. Asumir los deberes que se deben tener para cuidar la existencia. Identificar la ternura como una manifestación de afecto y de respeto. El Cuadro 1 presenta una síntesis del enfoque y los contenidos utilizados.

El desarrollo de la propuesta mostró las ventajas y desafíos que implican los cambios en los enfoques educativos y pedagógicos y la necesidad de fortalecer en los niños la posibilidad de participar de la familia, el Estado y la sociedad civil, en este sentido se precisa que:

- El proceso educativo es percibido como "lento", dado que la educación problematizadora, exige tiempo ya que se deben respetar los ritmos de aprendizaje de los participantes, quienes requieren asimilar la intencionalidad y racionalidad de la misma.

- La educación problematizadora confronta los conceptos del poder de los maestros o de quienes hagan las veces de facilitadores y requiere que la confianza y la libertad sean valores que faciliten el desarrollo de la autonomía de los participantes en el proceso, por tanto se debe ser muy persuasivo para lograr que se vinculen al proceso.

- La evaluación cualitativa favorece el aprendizaje y estimula la crítica, por lo que para los participantes en el proceso, los encuentros educativos exigieron permanente preparación, hecho que cualificó la participación.

- Se deben construir espacios de interacción que faciliten la libertad de expresión y la vivencia de valores como el respeto, la solidaridad y tolerancia, a partir de reconocer en cada uno de los participantes a una persona integral, comprendida como un ser biológico, psicológico, social, ético, político, estético, cultural, histórico, productivo y espiritual, por lo que cada persona tiene un saber aprendido producto de los procesos de socialización en los diferentes espacios que son permeados por una cultura que sustentan la importancia de asumir la diferencia como una posibilidad de éxito y no como una dificultad.

- Los temas de sexualidad requieren el conocimiento y apoyo de la familia, por lo que debe ser enfocada como expresión de vida, con una reflexión intensa acerca de las actitudes asumidas por los participantes al iniciar la temática, resaltando la importancia de fortalecer la aceptación del cuerpo, la interacción entre géneros y la tolerancia • 


\section{REFERENCIAS}

1. Colombia, Presidencia de la República. Decreto 1210 de 1993. Diario Oficial No. 40928, del 28 de junio de 1993

2. Gómez RD. La Noción de la Salud Pública consecuencias de la polisemia. Rev Fac Nac Salud Pública. 2002; 20(1):101-16.

3. Gómez H. Incorporación del concepto de ética social dentro del concepto de ética médica. Anales de la Facultad de Medicina Universidad Nacional Mayor de San Marcos.1967; 50(2). [Internet]. Disponible en: http://sisbib.unmsm.edu.pe/ bvrevistas/anales/v50_n2/indexa.htm. Consultado Marzo 2011.

4. Colombia, Congreso de la República. Ley 266 De 1996. (Enero 25). Diario Oficial No. 42.710, del 5 de febrero de 1996

5. De Erice MVG. Pequeño Glosario de Semántica. Universidad Nacional del Cuyo; 2010 [updated. 2010; cited 201024 de septiembre]; Available from: http://es.scrbd.com/ doc/4969603/ Glosario-de-Semantica.

6. Max-Neef M. Desarrollo a escala humana. Conceptos, aplicaciones y reflexiones. Santiago de Chile: Icaria Antrazyt; 1993.

7. Organización Panamericana de la Salud. Funciones Esenciales de Salud Pública (FESP). Washington: PAHO; 2010 [Internet]; Consultado 2010 junio 24; Disponible: http:// www.paho .org/Spanish/DPM/SHD/HP/FESP.htm.

8. Pedroza ST, Gutiérrez R. Los niños y niñas como grupo vulnerable: una perspectiva constitucional In: Valadés D, Gutiérrez Rivas R, editores. Derechos Humanos Memoria del IV Congreso Nacional de Derecho Constitucional III México: Instituto de estudios Jurídicos. Universidad Nacional Autónoma de México; 2001. p.103-23.

9. Ramírez H. Estrategia de Escuela Saludable. Material impreso. Medellín: Universidad de Antioquia; 2003.

10. Colombia, Congreso de la República. Ley General de la educación, Ley 115. De 1994. (febrero 8). Diario Oficial No. 41.214, de 8 de febrero de 1994

11. Freire P. Pedagogía del Oprimido. México: Siglo Veintiuno; 1999.

12. Palomo AM. Kohlberg L. Teoría y práctica del desarrollo moral en la escuela. . Rev Interuniv Form Profr. 1989; (4):79-90.

13. Asamblea Naciones Unidas. Convención sobre los derechos del niño., Resolución 44/25. (1989).

14. Vygotski LS. El desarrollo de los procesos psicológicos superiores. 3a ed. Barcelona: Crítica; 2009.

15. Cortina, A. Ética mínima, Madrid, Tecnos; 1986

16. Puerto AH. Servicio de Enfermería para el Cuidado de la Vida y la salud de la niñez en la escuela saludable. Bogotá: Universidad Nacional de Colombia (Sede Bogotá). Facultad de Enfermería; 2004.

17. República de Colombia. Proyecto Nacional de educación sexual para la vida y el amor. Bogotá: Ministerio de Educación Nacional; 1998.

18. Osorio PA. Desarrollo Familiar una Alternativa al Cambio Familiar y Social. Grupo Motivador de Estudiantes, Universidad de Caldas. [Internet]. Consultado 08 cited 2010 Agosto 28: Disponible: http://200.21.104.25/grume/investigacion/index.php?v iew=article \&catid=27\%3Aarticulos\&id=108\%3Adesarrollo-familiar-una-alternativaal-cambio-familiar-y-social\&option=com_content\&ltemid=38.

19. Lamus D. Representaciones Sociales de Maternidad y Paternidad en Cinco Ciudades Colombianas. Reflexión Política. 1999; 1(2):1-11.

20. Alcaldía Mayor de Bogotá. Plan de Salud del Distrito Capital. Bogotá: Secretaria Distrital de Salud; 2008.

21. Sen A. Desarrollo y Libertad. 4ta ed. Bogotá: Editorial Planeta Colombiana S.A; 2002. 